\title{
Experimental and numerical study on fragmentation of steel projectiles
}

\author{
K.G. Rakvåg ${ }^{1, a}$, T. Børvik ${ }^{1,2}$, O.S. Hopperstad ${ }^{1}$, and I. Westermann ${ }^{3}$ \\ 1 Structural Impact Laboratory (SIMLab), Centre for Research-based Innovation (CRI) and Department \\ of Structural Engineering, Norwegian University of Science and Technology, Rich. Birkelandsvei 1A, \\ 7491 Trondheim, Norway \\ 2 Norwegian Defence Estates Agency, Research \& Development Department, PB 405, Sentrum, 0103 Oslo, \\ Norway \\ 3 SINTEF Materials and Chemistry, Rich. Birkelandsvei 2B, 7465 Trondheim, Norway
}

\begin{abstract}
A previous experimental study on penetration and perforation of circular Weldox 460E target plates with varying thicknesses struck by blunt-nose projectiles revealed that fragmentation of the projectile occurred if the target thickness or impact velocity exceeded a certain value. Thus, numerical simulations that do not account for fragmentation during impact can underestimate the perforation resistance of protective structures. Previous numerical studies have focused primarily on the target plate behaviour. This study considers the behaviour of the projectile and its possible fragmentation during impact. Hardened steel projectiles were launched at varying velocities in a series of Taylor tests. The impact events were captured using a high-speed camera. Fractography of the fragmented projectiles showed that there are several fracture mechanisms present during the fragmentation process. Tensile tests of the projectile material revealed that the hardened material has considerable variations in yield stress and fracture stress and strain. In the finite element model, the stress-strain behaviour from tensile tests was used to model the projectile material with solid elements and the modified Johnson-Cook constitutive relation. Numerical simulations incorporating the variations in material properties are capable of reproducing the experimental fracture patterns, albeit the predicted fragmentation velocities are too low.
\end{abstract}

\section{Introduction}

In this study, the classical Taylor test is used to test and provoke fragmentation. The test was originally conceived in 1948 by Taylor [1] as a material test to determine the dynamic compressive yield stress of the material. The test procedure is to impact a blunt-nose projectile into a rigid wall, and based on post-impact measurements of the deformed cylinder, material properties are extracted based on Taylor's analysis. It has later been shown that the Taylor test is not suitable as a material characterization test. The results from the Taylor analysis are at best approximations, and it has been shown through experiments that the analysis does not predict the deformation or derive the yield strength accurately [2]. Hence, the Taylor test is used herein as a component test, and tension tests are used as material tests. Well over 400 scientific articles have been published with relation to the Taylor test [3], but only a few have investigated fracture or fragmentation in the projectile. Woodward et al. [4] identified four distinct modes of failure in the projectile during the Taylor test for high-strength steel:

1. Tensile splitting on the edge of the mushroomed end due to hoop strain exceeding the failure strain without fragments.

2. Shear fracture where a conical region separates.

3. Combined spiral shear fracture and tensile splitting where the mushroomed material separates from the impact end of the projectile.

4. Void nucleation due to hydrostatic tension in the centre of the projectile.

a e-mail: knut.gaarder.rakvag@ntnu.no
Teng et al. [3] attempted to predict fracture in numerical simulations of the Taylor test using element erosion. Different ductile fracture criteria were then compared to see how well they would model the various deformation mechanisms. They identified a possible fifth mode of failure in the numerical simulations; sunflower-like petalling for the relatively ductile Weldox 460E steel.

Chen et al. [5] performed a study on soft steel projectiles impacting a harder, but not rigid, target plate with velocities ranging from $200-800 \mathrm{~m} / \mathrm{s}$. They experimentally identified the sunflower-like petalling mode predicted by Teng et al. They also noted an important difference between the Taylor test and their own nonpenetrating experiments. With a deformable target plate the mushroomed part of the impact end consists of two parts: An inner circle with diameter approximately equal to the undeformed projectile diameter and an outer loop of material. The tensile splitting in the outer loop never crossed the interface between the parts. They noticed that the reason for the petalling is that the outer loop separates from the inner circle.

Xiao et al. [6] performed experiments on both soft and hard projectiles. For the soft projectiles mushrooming, shear cracking and petalling were identified. The failure modes for the hard projectiles were mushrooming, shear cracking and fragmentation. For the mushrooming they experienced the same inner and outer loop pattern as Chen et al. [5] due to a small indentation in the target plate, even though the target plate was harder than the projectile. These results highlight another shortcoming of Taylor's analysis, that the target plate is rigid and remains a flat plane during the impact. The shear cracks in the soft projectiles stopped at the interface between the 
Table 1. Chemical composition of Arne tool steel.

\begin{tabular}{lllll}
\hline $\mathrm{C}$ & $\mathrm{Mn}$ & $\mathrm{Cr}$ & $\mathrm{W}$ & $\mathrm{V}$ \\
\hline 0.95 & 1.1 & 0.6 & 0.6 & 0.1 \\
\hline
\end{tabular}

two loops, supporting a triaxility cut-off for the fracture criterion proposed by Teng et al. [3], as the outer loop is in tension in the hoop direction and the inner part is in compression due to the momentum of the projectile tail. However, in the hard projectiles the shear cracks crossed the interface and generated some loose fragments and a pointed projectile nose as experienced by Woodward et al. [4]. Also, based on numerical simulations Xiao et al. [7] speculate that the deformation mechanisms for shear cracking and fragmentation are the same.

Meyer and Brannon [8] used a Weibull distribution of the fracture properties in simulations of a Behind Armor Debris experiment. They found that the statistical failure model predicted the size distribution of fragments better than a homogenous failure model. They argue that this is due to the inherent variation of the material properties in the bulk material.

The scope of this paper is to investigate whether the critical velocity for fragmentation or fracture of the projectile can be identified. When the projectile fragments, it loses much of its penetrating capacity and thus this critical velocity is important for design of protective structures.

\section{Experimental study}

\subsection{Material tests}

The projectiles used in the Taylor impact tests were made of Arne tool steel hardened to a nominal Rockwell C value of 52. The alloying contents are listed in Table 1, and similar projectiles have been used in previous impact tests, e.g. [9] [10]. Axisymmetric specimens with a gauge length of about $40 \mathrm{~mm}$ and diameter of $6.0 \mathrm{~mm}$ were spark eroded from hardened projectiles. Three parallel tests were carried out at room temperature. The crosshead velocity of the tension machine during testing was $1.5 \mathrm{~mm} / \mathrm{min}$, which corresponds to an average strain rate in the gauge area of $6 \times 10^{-4} \mathrm{~s}^{-1}$. The force and the diameter at minimum cross-section of the specimen were continuously measured until fracture. The latter was made possible using a purpose-built measuring rig with two perpendicular lasers that accurately measured the specimen diameter. The lasers were installed on a mobile frame to ensure that the diameter during straining always was measured at minimum cross-section. The Cauchy (true) stress and the logarithmic (true) plastic strain were calculated as

$$
\sigma=\frac{F}{A}, \quad \epsilon^{p}=\ln \frac{A_{0}}{A}-2 v \frac{\sigma}{E}
$$

where $F$ is the force, $A_{0}=\frac{\pi}{4} D_{0}^{2}$ is the initial cross-section area, $D_{0}$ is the initial diameter of the gauge section, $v$ is the Poisson ratio and $E$ is the Young modulus. Due to possible variations in stress and strain over the cross-section, $\sigma$ and $\epsilon$ should be considered as average values. The current area

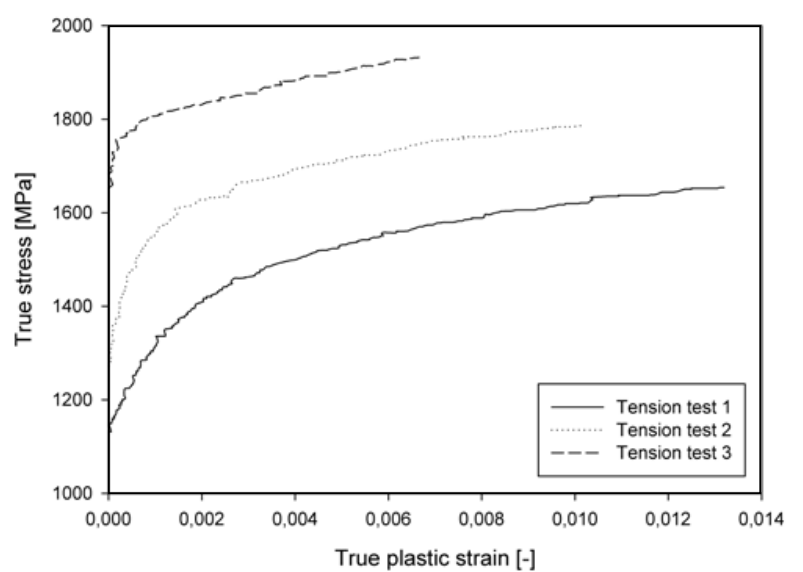

Fig. 1. True stress versus true plastic strain in uniaxial tension for Arne tool steel hardened to HRC 52.

of the cross-section is

$$
A=\frac{\pi}{4} D_{1} D_{2}
$$

where $D_{1}$ and $D_{2}$ are the diameters as measured by the lasers. Note that the measured Cauchy stress $\sigma$ is equal to the von Mises equivalent stress $\bar{\sigma}$ in the uniaxial tension test because the specimens fractured before the onset of necking. The Cauchy stress - logarithmic plastic strain curves until fracture are shown in Figure 1. The yield stress, the peak stress and the fracture strain varies considerably between parallel tests. Dynamic tensile tests on unhardened specimens were also conducted in a splitHokinson tension bar (SHTB). In these tests the strain rate was varied between 100 and $1000 \mathrm{~s}^{-1}$. The strain-rate sensitivity of the Arne tool steel was found to be rather low. Since it is assumed that the strain-rate sensitivity decreases with increasing hardness, the possible effects of strain-rate sensitivity will be neglected in this study.

\subsection{Taylor impact tests}

A compressed gas gun was used to accelerate the sabotmounted projectiles, and the impact process was recorded by a high-speed camera. Laser curtains were used to measure the impact velocities and trigger the high-speed camera. The cylindrical and blunt-nosed projectiles had nominal length $80 \mathrm{~mm}$, diameter $20 \mathrm{~mm}$, mass $197 \mathrm{~g}$ and hardness HRC 52, and they impacted a cylindrical target plate with diameter $100 \mathrm{~mm}$, thickness $50 \mathrm{~mm}$ and hardness HRC 60 . The target plate was firmly attached to a larger support steel plate with thickness $15 \mathrm{~mm}$, which in turn was bolted to a rigid frame. The distance from the muzzle of the barrel to the target plate was about $2.0 \mathrm{~m}$.

Here, only a few test results in the impact velocity range where the projectile behaviour changed from plastic deformation (mushrooming) to fragmentation will be presented. Figure 2 shows high-speed camera images of the projectiles immediately after impact at velocities of 124 , 135 and $154 \mathrm{~m} / \mathrm{s}$. At an impact velocity of $124 \mathrm{~m} / \mathrm{s}$ and below, the projectile is intact after impact and only slightly plastically deformed. At an impact velocity of $135 \mathrm{~m} / \mathrm{s}$, the projectile fractured in a shear-like failure mode at a 


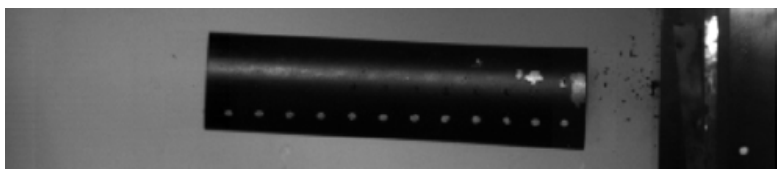

(a)

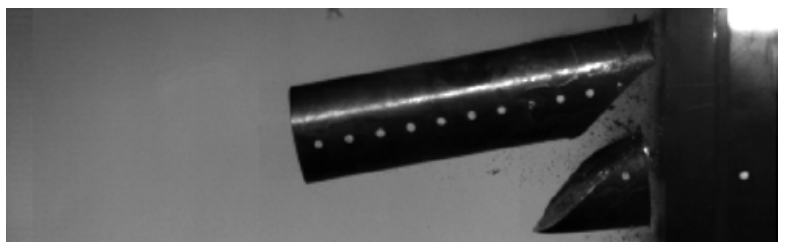

(b)

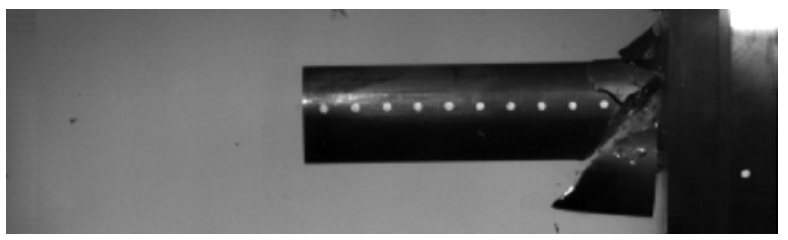

(c)

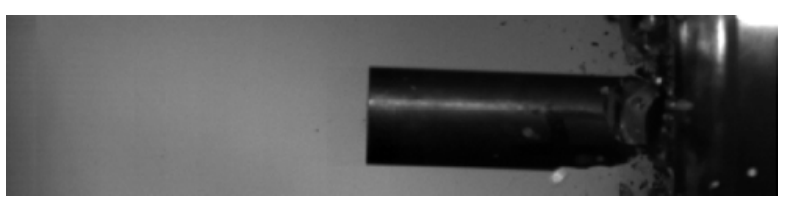

(d)

Fig. 2. High speed camera photographs of the projectiles after impact with the target plate for velocites ranging from 124.4 to $153.7 \mathrm{~m} / \mathrm{s}$. (a) $124.4 \mathrm{~m} / \mathrm{s}$; (b) $132.9 \mathrm{~m} / \mathrm{s}$; (c) $134.7 \mathrm{~m} / \mathrm{s}$ and (d) $153.7 \mathrm{~m} / \mathrm{s}$.

major plane $\approx 45^{\circ}$ to the longitudinal axis of the projectile. At an impact velocity of $154 \mathrm{~m} / \mathrm{s}$ and above, the projectile shattered into a number of fragments.

The experimental results presented in Figure 2 is an excerpt from a larger test series with velocities ranging from 120 to $300 \mathrm{~m} / \mathrm{s}$. An ongoing metallurgical investigation of the fragmented projectiles indicates that projectiles that fractured at higher velocities have a primary shear fracture similar to the one observed in Figure 2(b) and secondary cleavage fracture which is responsible for the fragmentation. An excerpt of the fractography that was obtained from a hardened projectile that fragmented at $200 \mathrm{~m} / \mathrm{s}$ is shown in Figure 3.

The variety of fracture surfaces present in the fragments from the projectile, as shown in Fig. 3, implicate that there are several fracture mechanisms active in the fragmentation process. The fracture surface in Figure 3(a) shows a typical quasi-cleavage fracture with river patterns (cleavage), but also evidence of ductile behaviour (i.e. voids). This fracture surface is from a fragment that is away from the front end. Figure 3(b) clearly shows what might be melted material, possible due to extreme temperatures in the shear zone. Figure 3(c) shows the brittle surface of the primary shear fracture. The variety in fracture modes complicates the fracture modelling both in terms of choice of method and input parameters. Thus, the focus of the numerical study will be to recreate a shear fracture similar to the one shown in Figure 2(b).

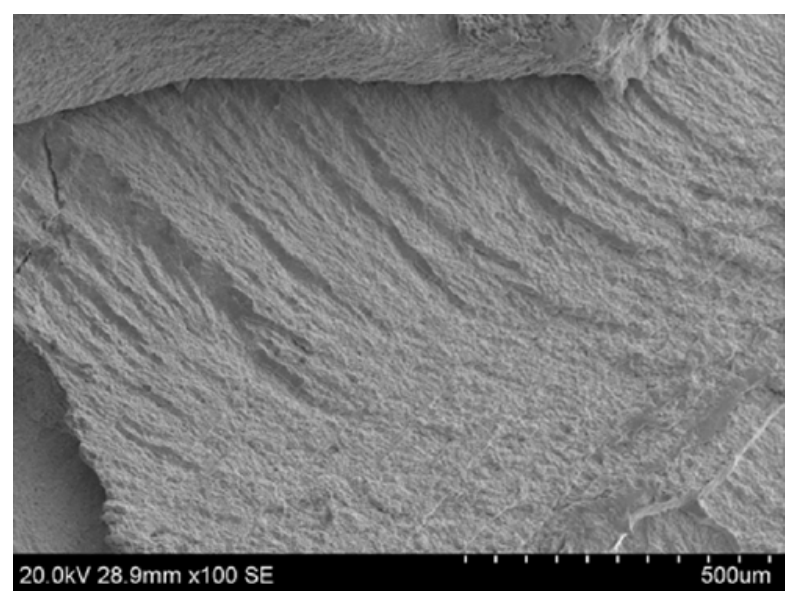

(a)

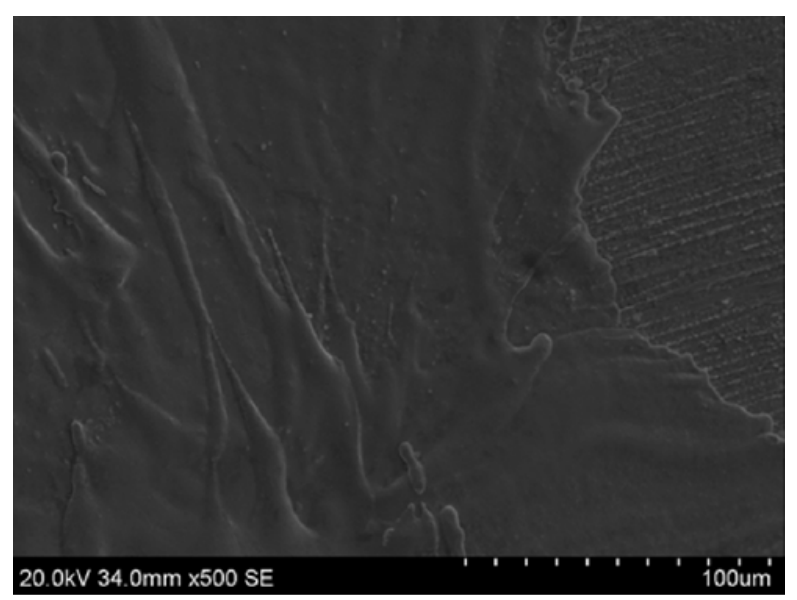

(b)

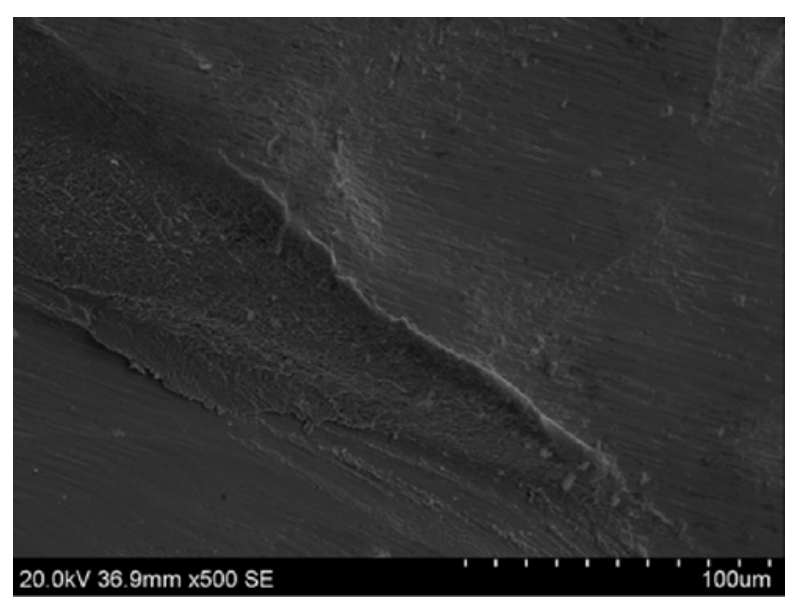

(c)

Fig. 3. SEM fractography of a fragmented projectile showing different fracture surfaces in the projectile. (a) Quasi-cleavage; (b) Possible melted material and (c) Brittle fracture in the shear zone.

\section{Numerical study}

The main intension with the numerical study was to try to capture the transition in projectile behaviour from plastic deformation to fragmentation during impact. In the finite element method, fracture is usually described by element 
Table 2. Nominal thermoelastic-thermoplastic material properties for steel.

\begin{tabular}{llllll}
\hline$\rho\left[\mathrm{kg} / \mathrm{m}^{3}\right]$ & $E[\mathrm{MPa}]$ & $v$ & $\chi$ & $C_{p}[\mathrm{~J} / \mathrm{kgK}]$ & $T_{m}[\mathrm{~K}]$ \\
\hline 7850 & 210000 & 0.3 & 0.9 & 452 & 1743 \\
\hline
\end{tabular}

erosion, cohesive zones models or node splitting. Cohesive zone modelling was tried as a first approach, including a thorough fracture mechanics test program consisting of dynamic Charpy testing and quasi-static three-point bending tests. This approach turned out to be unsuccessful, partly due to difficulties and uncertainties with the fracture testing. Problems were also encountered with the cohesive models as implemented in LS-DYNA. Thus, element erosion was chosen because of its simplicity. Element erosion also eliminates the need of establishing a fracture pattern from the outset, which is needed for cohesive zone modelling. Node splitting was not considered here, as it is in general not available for 3D simulations in commercial FE codes.

The projectile material was modelled with solid elements using the modified Johnson-Cook constitutive relation with Voce hardening and the Cockcroft-Latham failure criterion [11], and the true stress-strain data from the tensile tests (Figure 1) was used to obtain the material parameters given in Table 3. Nominal thermoelasticthermoplastic material properties for the steel used are shown in Table 2. The von Mises equivalent stress $\bar{\sigma}$ as a function of equivalent plastic strain $\bar{\epsilon}$ is expressed as

$$
\bar{\sigma}=\left[\sigma_{0}+\sum_{i=1}^{2} Q_{i}\left(1-\mathrm{e}^{\left(-C_{i} \bar{\epsilon}\right)}\right)\right]\left[1+\frac{\dot{\bar{\epsilon}}}{\dot{\overline{\epsilon_{0}}}}\right]^{c}\left[1-\left(T^{*}\right)^{m}\right]
$$

where $\dot{\bar{\epsilon}}$ is the equivalent plastic strain rate and $\dot{\overline{\epsilon_{0}}}$ is a userdefined reference strain rate. The constant $\sigma_{0}$ defines the initial yield strength of the material, whereas $Q_{i}$ and $C_{i}$, $i=1,2$, define the strain hardening. The homologoeus temperature is defined as

$$
T^{*}=\frac{T-T_{r}}{T_{m}-T_{r}}
$$

where $T$ is the absolute temperature, $T_{r}$ is the ambient temperature and $T_{m}$ is the melting temperature. The rate sensitivity is governed by the constant $c$, while $m$ defines the thermal softening behaviour. In this study $c$ is set equal to zero due to the low strain rate sensitivity of the base material, and $m$ is set to unity. The temperature change due to adiabatic heating may be calculated as

$$
\Delta T=\int_{0}^{\bar{\epsilon}} \chi \frac{\bar{\sigma}}{\rho C_{p}} \mathrm{~d} \bar{\epsilon}
$$

where $\rho$ is the material density, $C_{p}$ is the specific heat and $\chi$ is the Taylor-Quinney coefficient that represents the proportion of plastic work converted into heat. Finally, an element is eroded (all stresses set equal to zero) when

$$
D=\frac{1}{W_{c}} \int_{0}^{\bar{\epsilon}}\left\langle\sigma_{1}\right\rangle \mathrm{d} \bar{\epsilon}=1
$$

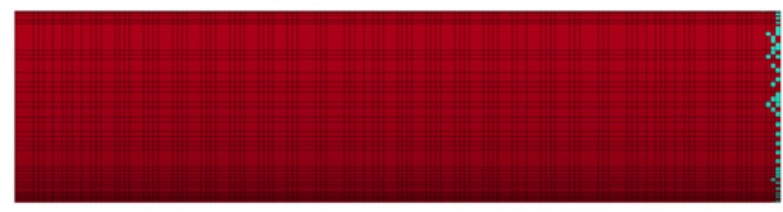

(a)

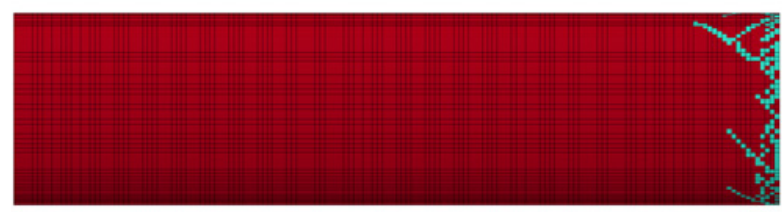

(b)

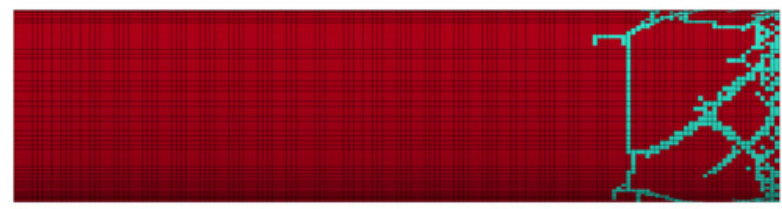

(c)

Fig. 4. Typical fracture pattern evolution in projectile simulated with homogeneous material. Deleted elements are shown on the undeformed configuration. (a) $110 \mathrm{~m} / \mathrm{s}$; (b) $115 \mathrm{~m} / \mathrm{s}$ and (c) $120 \mathrm{~m} / \mathrm{s}$.

where $D$ is the damage variable, $\left\langle\sigma_{1}\right\rangle=\max \left(\sigma_{1,0}\right)$ is positive values of the maximum principal stress and $W_{c}$ is the fracture parameter.

In the numerical analyses a characteristic element size of $0.5 \times 0.5 \times 0.5 \mathrm{~mm}^{3}$ was used, which gives a total of 240000 elements in the projectile. A contact formulation that allows for self-contact between newly created surfaces (i.e. the two surfaces of a propagating crack) is used both as contact between the projectile and the target plate and as self-contact in the fragmenting projectile. The target plate is modelled as a purely elastic steel. In the experiments, no indentation was found in the target plates at the velocities presented here, so it is reasonable to assume that the yield stress of the target plate was not exceeded.

\subsection{Numerical results}

As a first approach three sets of separate simulations with a homogeneous material from each tensile test were performed. Material parameters from tension tests 1 and 2 gave a fragmentation velocity of $120 \mathrm{~m} / \mathrm{s}$, while the parameters from tension test 3 gave a fragmentation velocity of $115 \mathrm{~m} / \mathrm{s}$. However, none of these simulations reproduced the shear fracture mode from the experiments. The fracture pattern evolution from $110 \mathrm{~m} / \mathrm{s}$ to $120 \mathrm{~m} / \mathrm{s}$ for the parameters from tension test 1 is shown in Figure 4.

As an alternative approach, a single projectile consisting of a random distribution of the material properties from each of the three tension tests was simulated. The distribution of material characteristics is shown in Figure 5 where red, blue and green in turn indicate material data from tensile test 1,2 and 3. 
Table 3. Material model parameters from the three tension tests.

\begin{tabular}{l|llllll}
\hline Test & $\sigma_{0}[\mathrm{MPa}]$ & $Q_{1}[\mathrm{MPa}]$ & $C_{1}$ & $Q_{2}[\mathrm{MPa}]$ & $C_{2}$ & $W_{c}[\mathrm{MPa}]$ \\
\hline 1 & 1134.4 & 959.3 & 18.3 & 324.0 & 690.3 & 23.0 \\
2 & 1285.2 & 1081 & 20.0 & 315.9 & 1652 & 18.7 \\
3 & 1660.4 & 1195 & 20.8 & 123.0 & 4591 & 14.5 \\
\hline
\end{tabular}

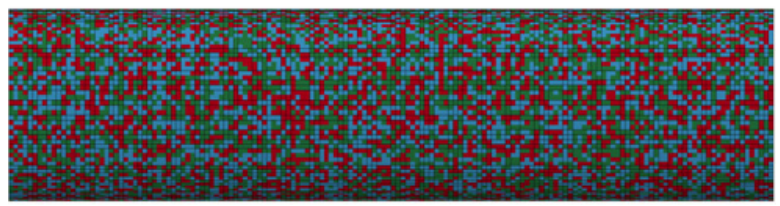

Fig. 5. Distribution of material parameters.

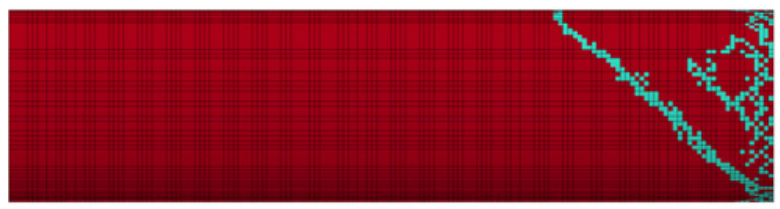

(a)

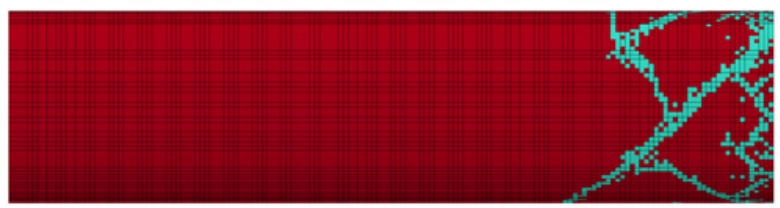

(b)

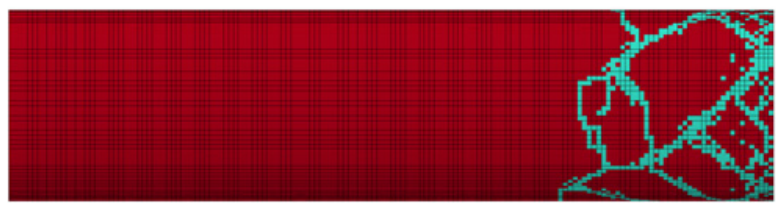

(c)

Fig. 6. Fracture pattern evolution in distributed projectile material. Deleted elements are shown on the undeformed configuration. (a) $110 \mathrm{~m} / \mathrm{s}$; (b) $115 \mathrm{~m} / \mathrm{s}$ and (c) $120 \mathrm{~m} / \mathrm{s}$.

This approach was capable of capturing the shear fracture pattern, although the fragmentation of the projectile happened at a lower velocity than in the previous analyses. Figure 6 shows the fracture pattern evolution of a projectile with randomly distributed material characteristics at a velocity ranging from $110 \mathrm{~m} / \mathrm{s}$ to $120 \mathrm{~m} / \mathrm{s}$.

Both the single shear fracture at $132.9 \mathrm{~m} / \mathrm{s}$ (Figure 2(b)) and the double shear fracture at $134.7 \mathrm{~m} / \mathrm{s}$ (Figure 2(c)) from the experiments are reproduced by this model, as well as the transition to fragmentation at the highest impact velocities. However, the critical velocity for projectile fracture is lower than the experimental velocities and also lower than for the simulations with a homogeneous material. The reason for this is probably the very crude way of distributing the material parameters, and a more thorough distribution should be implemented directly into the material model. A direct comparison of the primary shear fracture is shown in Figure 7, where it is seen that the numerical shear crack is very similar to the experimental

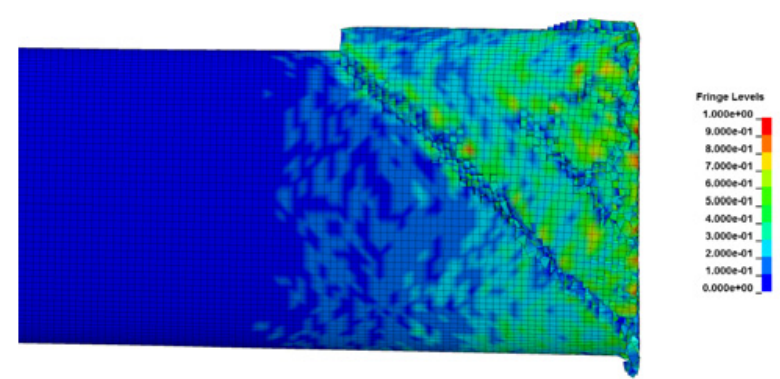

(a)

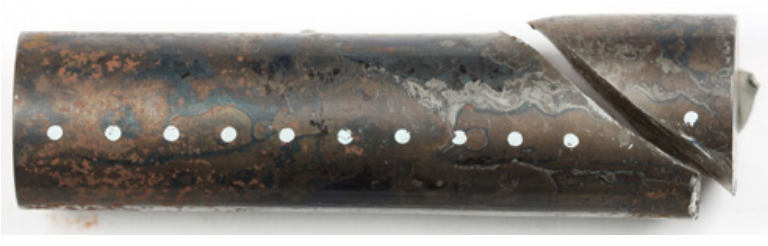

(b)

Fig. 7. Comparison of primary shear crack from simulations $(110 \mathrm{~m} / \mathrm{s})$ and experiments $(132.9 \mathrm{~m} / \mathrm{s})$. (a) Deformed configuration with fringes of the damage variable $D$ from numerical simulation at $110 \mathrm{~m} / \mathrm{s}$ and (b) Photo of experimental obtained shear crack at $132.9 \mathrm{~m} / \mathrm{s}$.

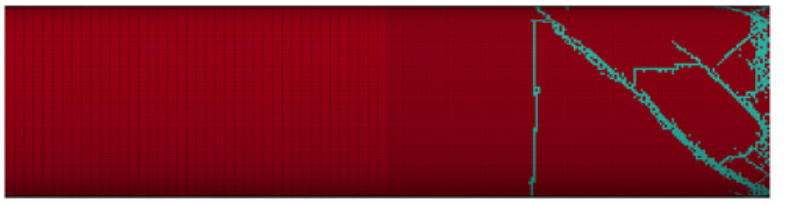

Fig. 8. Fracture pattern evolution in distributed projectile material with refined mesh. Deleted elements are shown on the undeformed configuration.

one, down to the initiation point and the lip at the end of the crack.

An important aspect to consider when using element erosion is the lack of an intrinsic length scale, which makes convergence unreliable [12]. Thus, the simulation with distributed material properties at $110 \mathrm{~m} / \mathrm{s}$ was carried out with an element size of $0.25 \times 0.25 \times 0.25 \mathrm{~mm}^{3}$ in the front half of the projectile, giving a total of 1.2 million elements. As shown in Figure 8 the primary shear crack is still reproduced, but several secondary cracks are now present. An interesting observation is that the maximum temperature increase $\Delta T$ from adiabatic heating in the projectile has risen from $530 \mathrm{~K}$ to $978 \mathrm{~K}$. The temperature increases considerably as the mesh is refined, but is still far from the melting temperature of steel. However, the possible melting of the material shown in Figure 3(b) takes place at a much lower scale than what is feasible in 
full-scale numerical simulations. Also the change in mass due to eroded elements is compared for the two analyses showing that the change is $5 \%$ for both analyses.

\section{Conclusion}

In this paper an experimental and numerical study on the fragmentation of hardened steel projectiles during impact has been presented. To provoke fragmentation, classical Taylor impact tests were carried out. Here, projectiles hardened to a Rockwell $\mathrm{C}$ value of 52 were fired into a hardened target plate, and the transition impact velocity from plastic deformation to fragmentation in the projectile was determined. Tensile tests were conducted to calibrate a proper constitutive relation and a fracture criterion in an attempt to numerically capture the fragmentation process. A metallurgical inspection of the fragments shows that there are many different fracture mechanisms present in the hardened material. It also indicates that the primary fracture is a shear fracture, supporting Xiao et al. hypothesis that the deformation mechanisms for shear cracking and fragmentation are the same [6]. A rather crude method to allow for the stochastic properties of the hardened material is shown to reproduce the shear crack pattern in a much better way than analyses with a homogeneous material. However, the fragmentation velocity is lowered by this approach, so further work is needed to develop a proper way to distribute the material parameters.

\section{References}

1. G. Taylor. Proc. R. Soc. Lond. A 194, 289-299 (1948)

2. W. Johnson. Impact Strength of Materials, (1972)

3. X. Teng, T. Wierzbicki, S. Hiermaier, I. Rohr. International Journal of Solids and Structures 42, (2005) 2929-2948

4. R.L. Woodward, R.G. ODonnell, C.J. Flockhart. Journal of Materials Science 27, (1992) 6411-6416

5. X.W. Chen, G. Chen, F.J. Zhang. Experimental Mechanics 48, (2008) 335-354

6. X. Xiao, W. Zhang, G. Wei, Z. Mu. Materials and Design 31, (2010) 4913-4920

7. X. Xiao, W. Zhang, G. Wei, Z. Mu, Z. Guo. Materials and Design 32, (2011) 2663-2674

8. H.W. Meyer Jr., R.M. Brannon. International Journal of Impact Engineering 42, (2012) 48-58

9. T. Børvik, O.S. Hopperstad, M. Langseth, K.A. Malo. International Journal of Impact Engineering 28, (2003) 413-464

10. T. Børvik, M. Langseth, O.S. Hopperstad, K.A. Malo. International Journal of Impact Engineering 22, (1999) 855-886

11. T. Børvik, O.S. Hopperstad, T. Berstad, M. Langseth. European Journal of Mechanics - A/Solids 20, (2001) 685-712

12. R. de Borst. Damage, Material Instabilities, and Failure (Encyclopedia of Computational Mechanics, 2004) 335-373 